TRANSACTIONS OF THE

AMERICAN MATHEMATICAL SOCIETY

Volume 363, Number 5, May 2011, Pages 2685-2700

S 0002-9947(2010)05210-9

Article electronically published on November 16, 2010

\title{
A DIAGRAMMATIC APPROACH TO CATEGORIFICATION OF QUANTUM GROUPS II
}

\author{
MIKHAIL KHOVANOV AND AARON D. LAUDA
}

\begin{abstract}
We categorify one-half of the quantum group associated to an arbitrary Cartan datum.
\end{abstract}

Cartan data and algebras ${ }_{\mathcal{A}}$ f. A Cartan datum $(I, \cdot)$ consists of a finite set $I$ and a symmetric bilinear form on $\mathbb{Z}[I]$ taking values in $\mathbb{Z}$, subject to the conditions

- $i \cdot i \in\{2,4,6, \ldots\}$ for any $i \in I$,

- $2 \frac{i \cdot j}{i \cdot i} \in\{0,-1,-2, \ldots\}$ for any $i \neq j$ in $I$.

We set $d_{i j}=-2 \frac{i \cdot j}{i \cdot i} \in \mathbb{N}$. To a Cartan datum assign a graph $\Gamma$ with the set of vertices $I$ and an edge between $i$ and $j$ if and only if $i \cdot j \neq 0$.

We recall the definition of the negative half of the quantum group associated to a Cartan datum, following [6. Let $q_{i}=q^{\frac{i \cdot i}{2}},[n]_{i}=q_{i}^{n-1}+q_{i}^{n-3}+\cdots+q_{i}^{1-n}$, $[n]_{i} !=[n]_{i}[n-1]_{i} \ldots[1]_{i}$. Let ' $\mathbf{f}$ be the free associative algebra over $\mathbb{Q}(q)$ with generators $\theta_{i}, i \in I$ and denote $\theta_{i}^{(n)}=\theta_{i}^{n} /[n]_{i}$ !. We equip 'f with an $\mathbb{N}[I]$-grading by assigning to $\theta_{i}$ grading $i$. The tensor square ' $\mathbf{f} \otimes{ }^{\prime} \mathbf{f}$ is an associative algebra with the multiplication

$$
\left(x_{1} \otimes x_{2}\right)\left(x_{1}^{\prime} \otimes x_{2}^{\prime}\right)=q^{-\left|x_{2}\right| \cdot\left|x_{1}^{\prime}\right|} x_{1} x_{1}^{\prime} \otimes x_{2} x_{2}^{\prime}
$$

for homogeneous $x_{1}, x_{2}, x_{1}^{\prime}, x_{2}^{\prime}$. There is a unique algebra homomorphism $r$ : 'f $\longrightarrow$ 'f $\mathbf{f} \otimes$ 'f given on generators by $r\left(\theta_{i}\right)=\theta_{i} \otimes 1+1 \otimes \theta_{i}$.

Proposition 1. Algebra' $\mathbf{f}$ carries a unique $\mathbb{Q}(q)$-bilinear form such that $(1,1)=1$ and

- $\left(\theta_{i}, \theta_{j}\right)=\delta_{i, j}\left(1-q_{i}^{2}\right)^{-1}$ for all $i, j \in I$,

- $\left(x, y y^{\prime}\right)=\left(r(x), y \otimes y^{\prime}\right)$ for $x, y, y^{\prime} \in{ }^{\prime} \mathbf{f}$,

- $\left(x x^{\prime}, y\right)=\left(x \otimes x^{\prime}, r(y)\right)$ for $x, x^{\prime}, y \in{ }^{\prime} \mathbf{f}$

This bilinear form is symmetric.

The radical $\mathfrak{I}$ of $($,$) is a two-sided ideal of 'ff. The bilinear form descends to a$ non-degenerate bilinear form on the associative $\mathbb{Q}(q)$-algebra $\mathbf{f}={ }^{\prime} \mathbf{f} / \mathfrak{I}$. The $\mathbb{N}[I]$ grading also descends:

$$
\mathbf{f}=\bigoplus_{\nu \in \mathbb{N}[I]} \mathbf{f}_{\nu}
$$

The quantum version of the Gabber-Kac theorem says the following.

Received by the editors June 6, 2009 and, in revised form September 9, 2009.

2000 Mathematics Subject Classification. Primary 81R50; Secondary 16 S99.

Key words and phrases. Categorification, quantum groups, Grothendieck ring, canonical basis.

The first author was fully supported by the IAS and the NSF grants DMS-0635607 and DMS0706924 while working on this paper.

(C)2010 American Mathematical Society Reverts to public domain 28 years from publication 
Proposition 2. The ideal $\mathfrak{I}$ is generated by the elements

$$
\sum_{a+b=d_{i j}+1}(-1)^{a} \theta_{i}^{(a)} \theta_{j} \theta_{i}^{(b)}
$$

over all $i, j \in I, i \neq j$.

Thus, $\mathbf{f}$ is the quotient of ' $\mathbf{f}$ by the so-called quantum Serre relations

$$
\sum_{a+b=d_{i j}+1}(-1)^{a} \theta_{i}^{(a)} \theta_{j} \theta_{i}^{(b)}=0
$$

Denote by ${ }_{\mathcal{A}} \mathbf{f}$ the $\mathbb{Z}\left[q, q^{-1}\right]$-subalgebra of $\mathbf{f}$ generated by the divided powers $\theta_{i}^{(a)}$, over all $i \in I$ and $a \in \mathbb{N}$.

Algebras $R(\nu)$. As in [4, we consider braid-like planar diagrams, each strand labelled by an element of $I$, and impose the following relations:

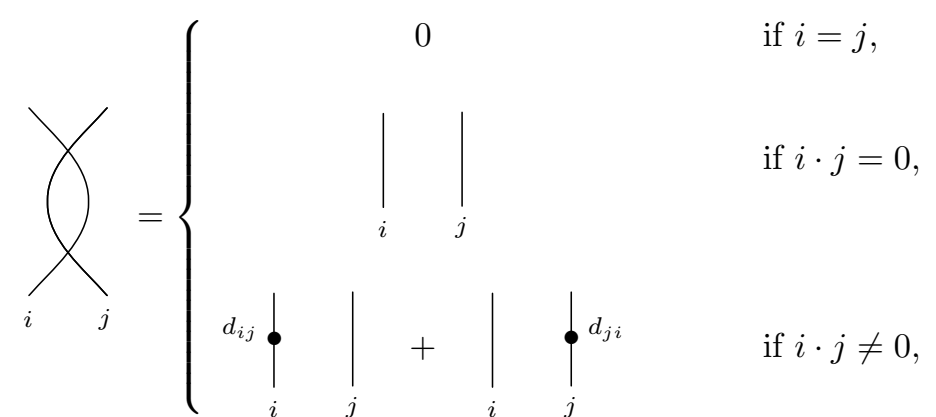

(3)
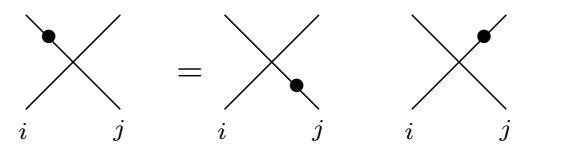

$=$

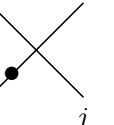

for $i \neq j$,

(6)
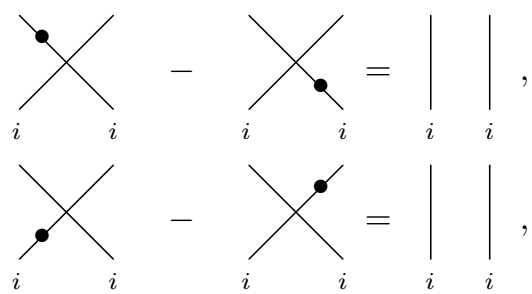

unless $i=k$ and $i \cdot j \neq 0$, 
$(7)$

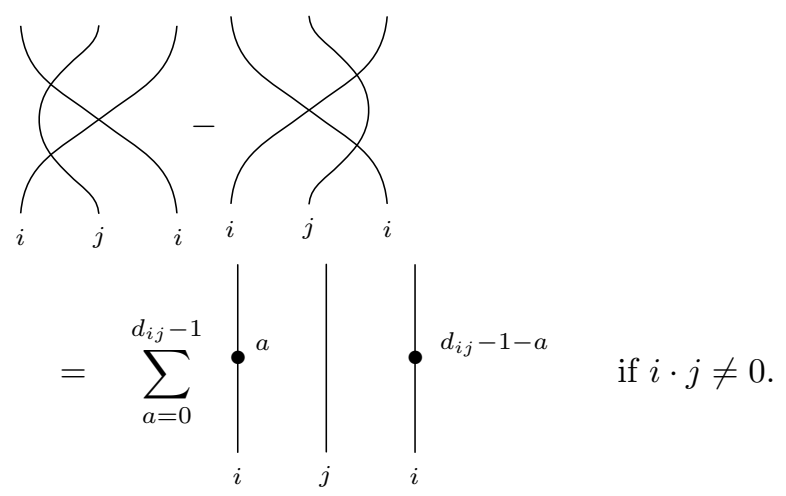

Example 3. For the Cartan datum $\mathbf{B}_{2}=\{i \cdot i=2, j \cdot j=4, i \cdot j=-2\}$ we have $d_{i j}=2, d_{j i}=1$, and the relations involving $d_{i j}, d_{j i}$ are

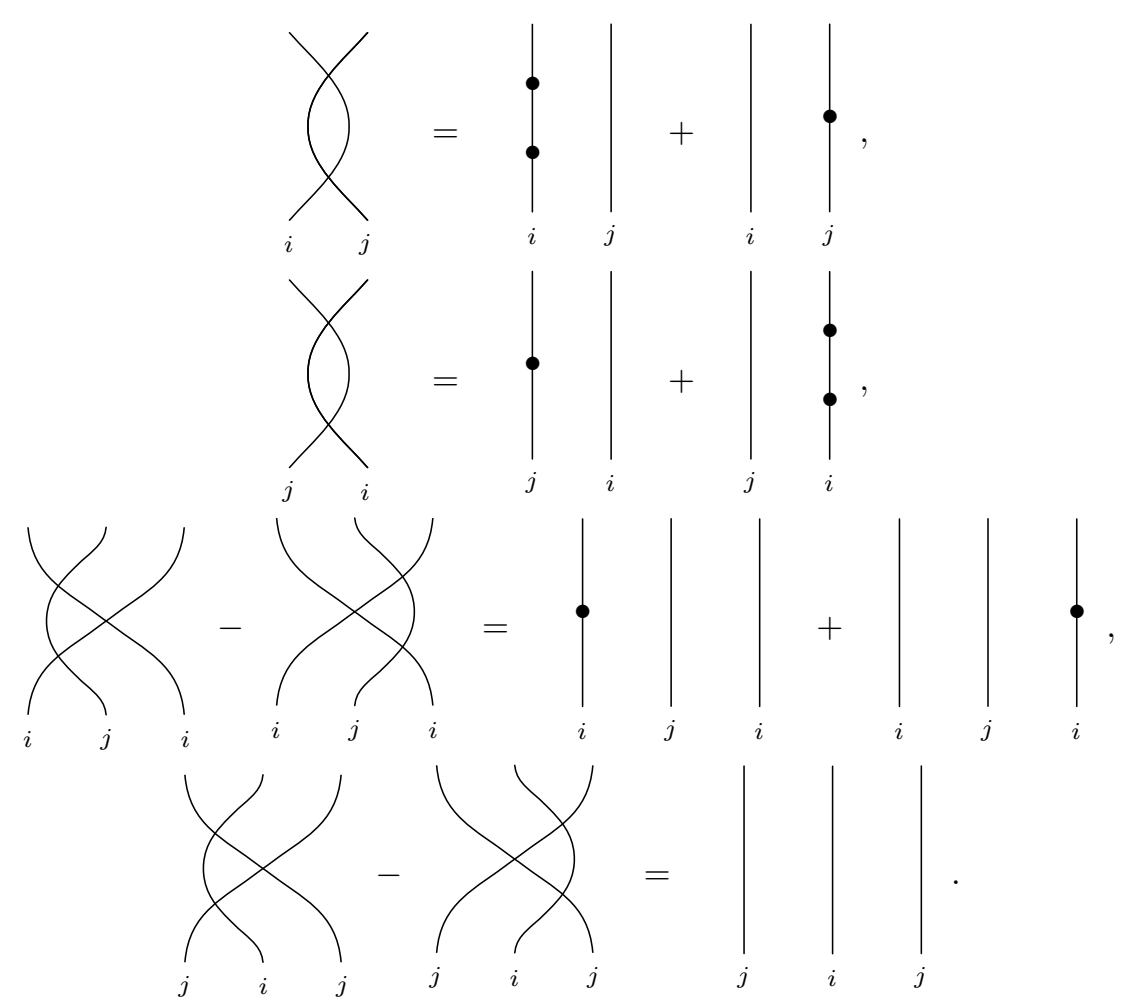

For each $\nu \in \mathbb{N}[I]$ define the graded ring

$$
R(\nu) \stackrel{\text { def }}{=} \bigoplus_{i, j \in \operatorname{Seq}(\nu)}{ }_{j} R(\nu)_{i}
$$

where ${ }_{j} R(\nu)_{i}$ is the abelian group of all linear combinations of diagrams with $\operatorname{bot}(D)=i$ and $\operatorname{top}(D)=j$ modulo the relations (2)-(7) and $\operatorname{Seq}(\nu)$ is the set 
of weight $\nu$ sequences of elements of $I$. The multiplication is given by concatenation. Degrees of the generators are:

$$
\operatorname{deg}\left(\prod_{i}\right)=i \cdot i, \quad \operatorname{deg}\left(\searrow_{i}\right)=-i \cdot j .
$$

The rest of [4, Section 2.1] generalizes without difficulty to an arbitrary Cartan datum. To define the analogue of the module $\mathcal{P}_{o} \ell_{\nu}$ over $R(\nu)$, we choose an orientation of each edge of $\Gamma$, then faithfully follow the exposition in Section 2.3 of [4, only changing the action of $\delta_{k, i}$ in the last of the four cases to

$$
f \mapsto\left(x_{k}\left(s_{k} \boldsymbol{i}\right)^{d}+x_{k+1}\left(s_{k} \boldsymbol{i}\right)^{d^{\prime}}\right)\left(s_{k} f\right) \quad \text { if } \quad i_{k} \longrightarrow i_{k+1},
$$

where $d=d_{i_{k+1} i_{k}}$ and $d^{\prime}=d_{i_{k} i_{k+1}}$. Here notation $i_{k} \longrightarrow i_{k+1}$ means that $i_{k} \cdot i_{k+1} \neq$ 0 and this edge of $\Gamma$ is oriented from $i_{k}$ to $i_{k+1}$. Proposition 2.3 in [4] holds for an arbitrary $(I, \cdot)$. As in [4, Section 2.3], we define ${ }_{j} B_{i}$, which might depend on minimal presentations of permutations in ${ }_{j} S_{i}$ and gives a basis in ${ }_{j} R(\nu)_{i}$. Corollary 2.6 in [4, showing that $\mathcal{P}_{o} \ell_{\nu}$ is a faithful graded module over $R(\nu)$, holds for an arbitrary Cartan datum and the properties of $R(\nu)$ established in [4, Section 2.4] generalize without difficulty.

Computations in the nil-Hecke ring. In this section we slightly enhance the graphical calculus for computations in the nil-Hecke ring and record several lemmas to be used in the proof of categorified quantum Serre relations below. We use notation from Section 2.2 of [4.

A box with $n$ incoming and $n$ outgoing edges and $\partial(n)$ written inside denotes the longest divided difference $\partial_{w_{0}}$, the non-zero product of $\frac{n(n-1)}{2}$ divided differences from $\left\{\partial_{1}, \ldots, \partial_{n-1}\right\}$ :

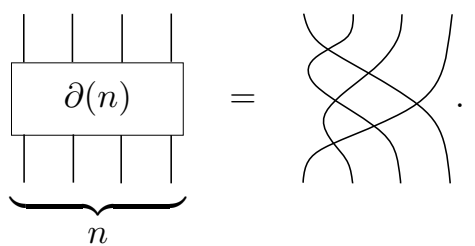

When this box is part of a diagram for an element of $R(\nu)$, it denotes the corresponding element of $R(n i) \subset R(\nu)$. A box labelled $e_{n}$ denotes the idempotent $e_{n}=x_{1}^{n-1} x_{2}^{n-2} \ldots x_{n-1} \partial_{w_{0}}$.

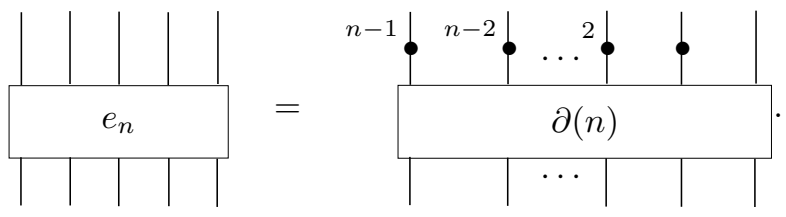


A box labelled $e_{i, n}$ denotes the corresponding idempotent in $R(\nu)$ :

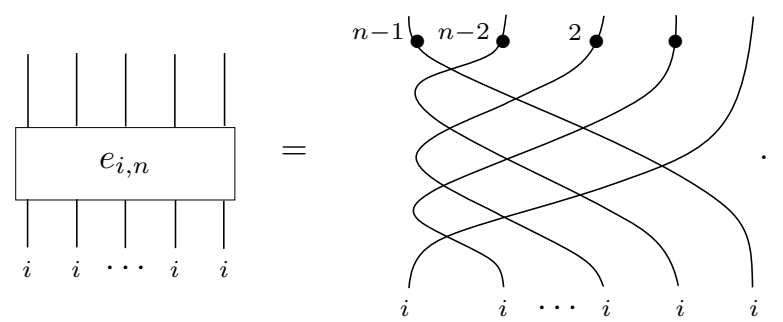

Remark 4. Similar diagrams are used in the graphical calculus of Jones-Wenzl projectors (see [3]), but the latter has no direct relation to the graphical calculus in our paper.

Lemma 5. We have

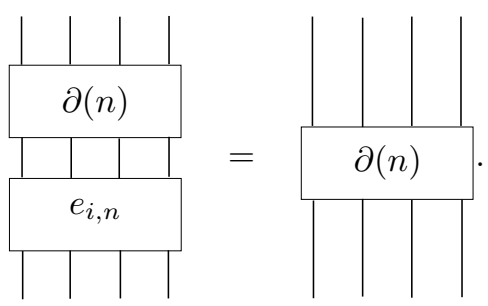

Proof is by induction on $n$ :

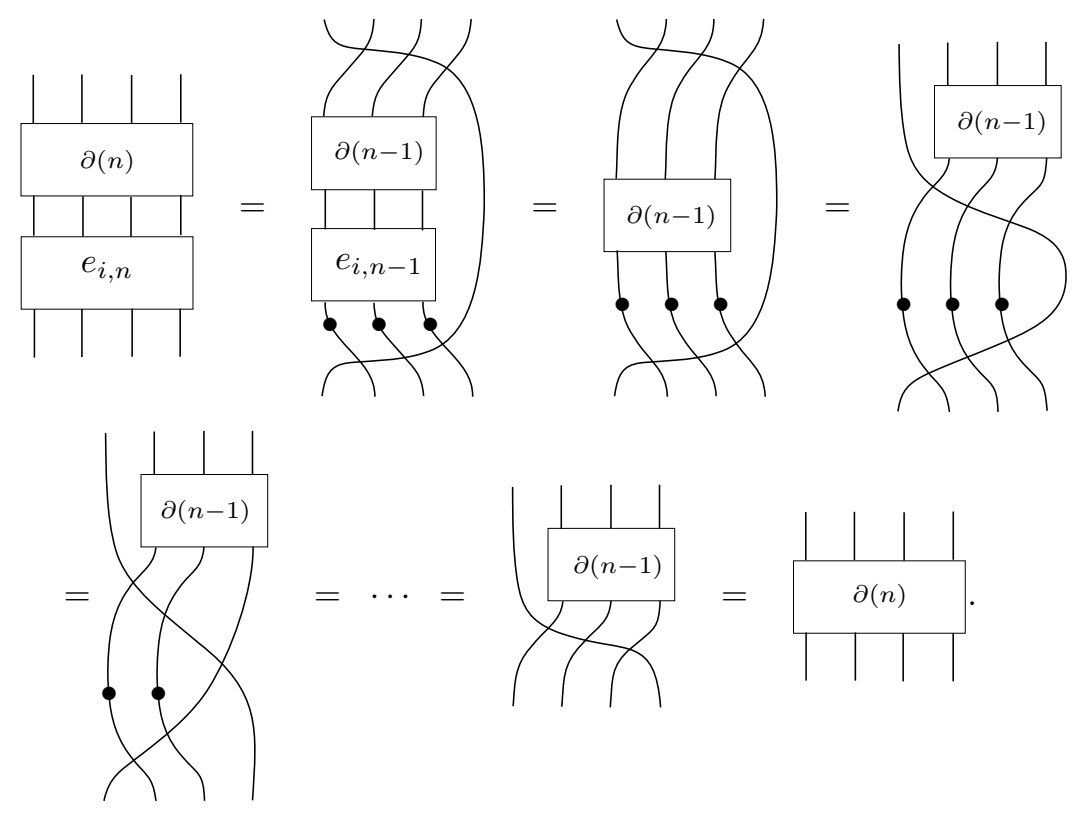

The first equality uses that $x_{1} x_{2} \ldots x_{n-1}$ is central in the nil-Hecke ring $N H_{n-1}$, allowing us to move these dots across $\partial(n-1)$. The second equality is the induction hypothesis. 
The lemma implies the following graphical identities:

(10)
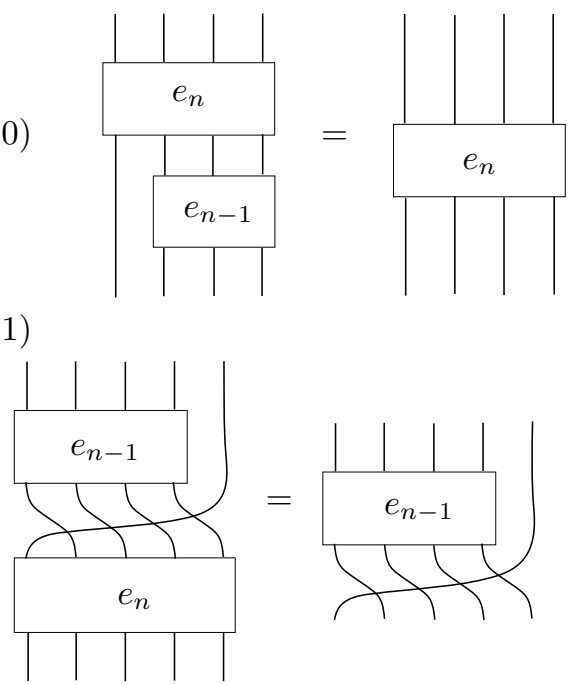
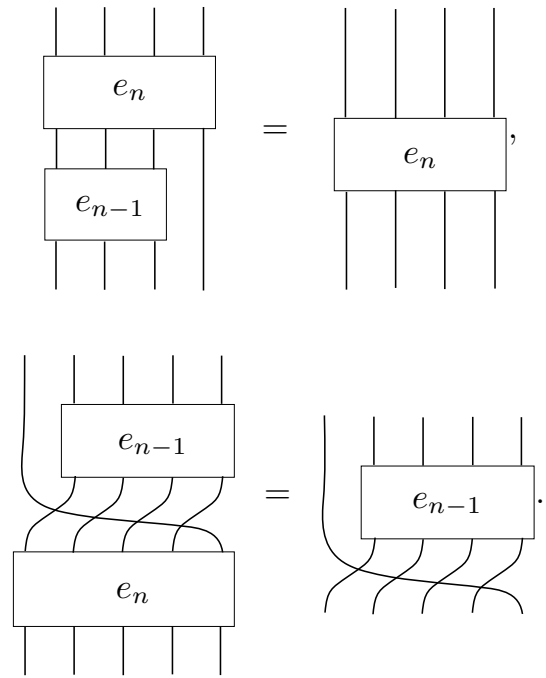

The following also hold:
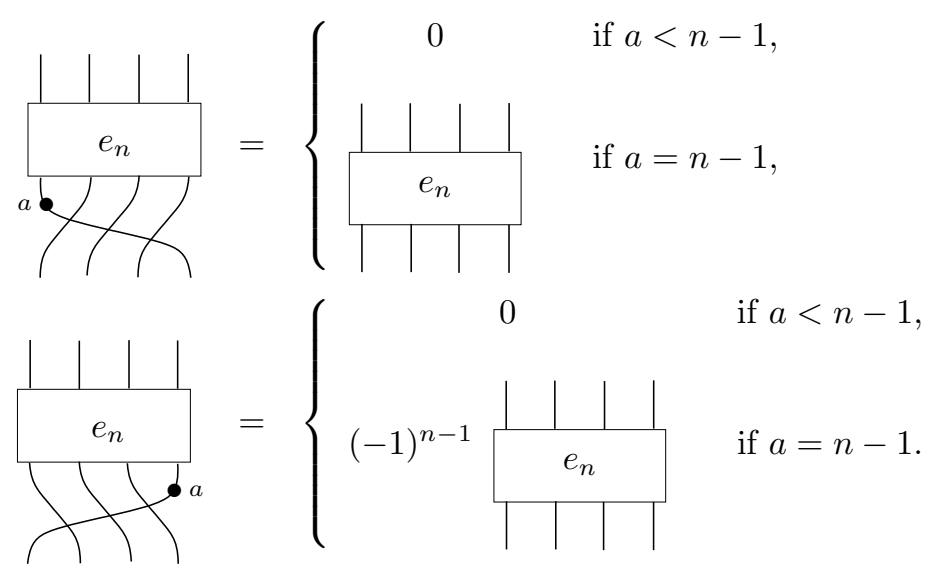

For each $i \in I$ the ring $R(m i)$ is isomorphic to the nil-Hecke ring. The grading of a dot is now $i \cdot i$, while that of a crossing is $-i \cdot i$. For this reason one needs to generalize the grading convention described in [4, Section 2.2] and define ${ }_{i, m} P$ to be the right graded projective module $e_{i, m} R(m i)\left\{-\frac{m(m-1) i \cdot i}{4}\right\}$, so that the grading starts in the degree $\left\{-\frac{m(m-1) i \cdot i}{4}\right\}$. Likewise, $P_{i, m}$ is the left graded projective module $R(m i) \psi\left(e_{i, m}\right)\left\{-\frac{m(m-1) i \cdot i}{4}\right\}$.

The Grothendieck group, bilinear form and projectives. We retain all notation and assumptions from [4, working over a field $\mathbb{k}$, denoting by $K_{0}(R(\nu))$ the Grothendieck group of the category $R(\nu)$-pmod of graded finitely-generated projective left $R(\nu)$-modules and forming the direct sum

$$
R=\bigoplus_{\nu \in \mathbb{N}[I]} R(\nu), \quad K_{0}(R)=\bigoplus_{\nu \in \mathbb{N}[I]} K_{0}(R(\nu))
$$


Consider symmetric $\mathbb{Z}\left[q, q^{-1}\right]$-bilinear form

$$
(,): K_{0}(R(\nu)) \times K_{0}(R(\nu)) \longrightarrow \mathbb{Z}\left[q^{-1}, q\right] \cdot(\nu)_{q},
$$

where

$$
(\nu)_{q}=\operatorname{gdim}(\operatorname{Sym}(\nu))=\prod_{i \in \Gamma}\left(\prod_{a=1}^{\nu_{i}} \frac{1}{1-q^{a i \cdot i}}\right)
$$

and

$$
([P],[Q])=\operatorname{gdim}_{\mathbb{k}}\left(P^{\psi} \otimes_{R(\nu)} Q\right) .
$$

The character $\operatorname{ch}(M)$ of an $R(\nu)$-module $M$, the divided power sequences $\operatorname{Seqd}(\nu)$, and idempotents $1_{i}$ for $i=i_{1}^{\left(n_{1}\right)} \ldots i_{r}^{\left(n_{r}\right)} \in \operatorname{Seqd}(\nu)$ are defined as in 4, Section $2.5]$. Let $i !=\left[n_{1}\right]_{i_{1}} ! \ldots\left[n_{r}\right]_{i_{r}} !$ and

$$
\langle\boldsymbol{i}\rangle=\sum_{k=1}^{r} \frac{n_{k}\left(n_{k}-1\right)}{2} \cdot \frac{i_{k} \cdot i_{k}}{2} .
$$

Define graded left, respectively right, projective module

$$
P_{i}=R(\nu) \psi\left(1_{i}\right)\{-\langle i\rangle\}, \quad{ }_{i} P=1_{i} R(\nu)\{-\langle i\rangle\} .
$$

Quantum Serre relations. Let

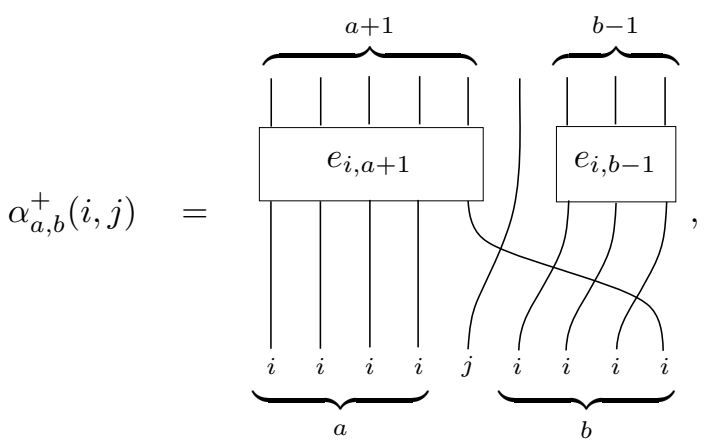

and also write $\alpha_{a, b}^{+}$when $i$ and $j$ are fixed. To prove the categorified quantum Serre relations, we assume that $a+b=d+1$, where $d=d_{i j}$. The element $\alpha_{a, b}^{+}$belongs to

$$
i^{a+1} j i^{b-1} R(j+(a+b) i)_{i^{a} j i^{b}} .
$$

By adding vertical lines on the left and on the right of the diagram, $\alpha_{a, b}^{+}$can be viewed, more generally, as an element of

$$
i^{\prime} i^{a+1} j i^{b-1} i^{\prime \prime} R(\nu)_{i^{\prime} i^{a} j i^{b} i^{\prime \prime}}
$$

for any sequences $\boldsymbol{i}^{\prime}, \boldsymbol{i}^{\prime \prime}$ and the corresponding $\nu$. We can replace sequences $\boldsymbol{i}^{\prime}$ and $i^{\prime \prime}$ by dots to simplify notation.

Left multiplication by $\alpha_{a, b}^{+}$is a homomorphism of projective modules

$$
\ldots i^{a} j i^{b} \ldots P \longrightarrow \ldots i^{a+1} j i^{b-1} \ldots P .
$$

The top part of the diagram of $\alpha_{a, b}^{+}$contains idempotents $e_{i, a+1}$ and $e_{i, b-1}$. Therefore, $\alpha_{a, b}^{+}$induces a homomorphism of projective modules

$$
\ldots i^{(a)} j i^{(b)} \ldots P \longrightarrow \ldots i^{(a+1)} j i^{(b-1)} \ldots P
$$


denoted $\alpha_{(a, b)}^{+}$and given by the composition

$$
\ldots i^{(a)} j i^{(b)} \ldots P C P_{\ldots i^{a} j i^{b} \ldots P} \stackrel{\alpha_{a, b}^{+}}{\longrightarrow} \ldots i^{(a+1)} j i^{(b-1)} \ldots P .
$$

It is easy to check that $\alpha_{(a, b)}^{+}$is a grading-preserving homomorphism. Likewise, let

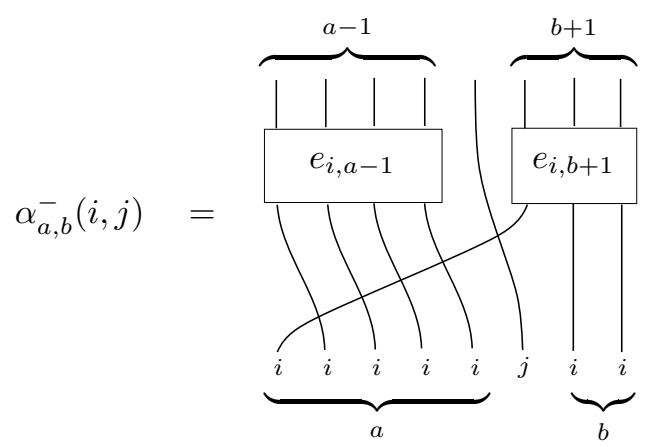

and write $\alpha_{a, b}^{-}$instead of $\alpha_{a, b}^{-}(i, j)$ when $i$ and $j$ are fixed. This element of

$$
\ldots i^{a-1} j i^{b-1} \ldots R(\nu) \ldots i^{a} j i^{b} \ldots
$$

gives rise to a grading-preserving homomorphism of projectives

$$
\alpha_{(a, b)}^{-}: \ldots i^{(a)} j i^{(b)} \ldots P \longrightarrow \ldots i^{(a-1)} j i^{(b+1)} \ldots P .
$$

For the next few pages, denote $\ldots i^{(a)} j i^{(b)} \ldots P$ by ${ }_{(a, b)} P$ (recall that $a+b=d+$ $1, d=d_{i j}$ ). We have a diagram of projective modules and grading-preserving homomorphisms

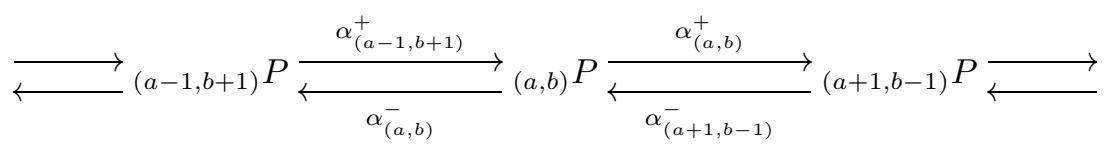

terminating on the left at

$$
{ }_{(0, d+1)} P \underset{\alpha_{(1, d)}^{-}}{\alpha_{(0, d+1)}^{+}}(1, d) P \longleftarrow
$$

and on the right at

$$
\cdots \varlimsup_{(d, 1)} P \underset{\alpha_{(d+1,0)}^{-}}{\longleftarrow}(d+1,0) P
$$


Relations (10), (11) imply
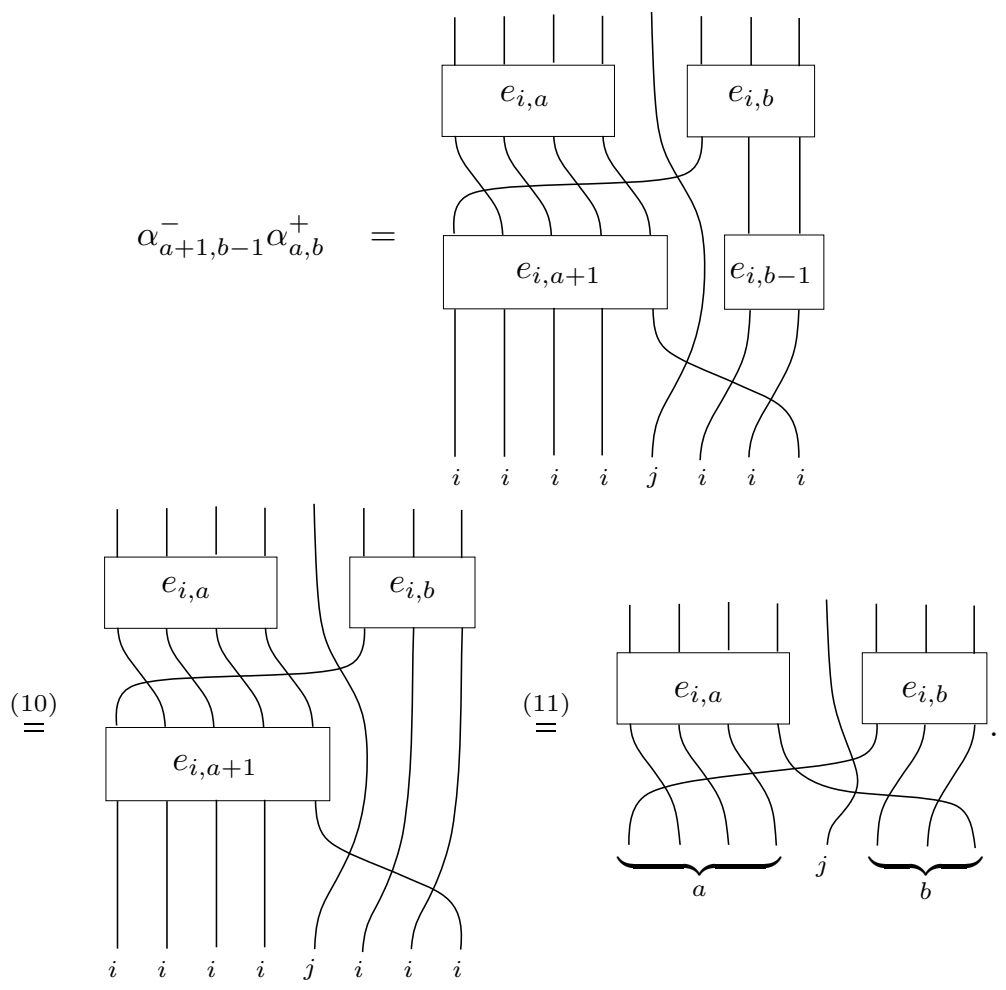

Furthermore,

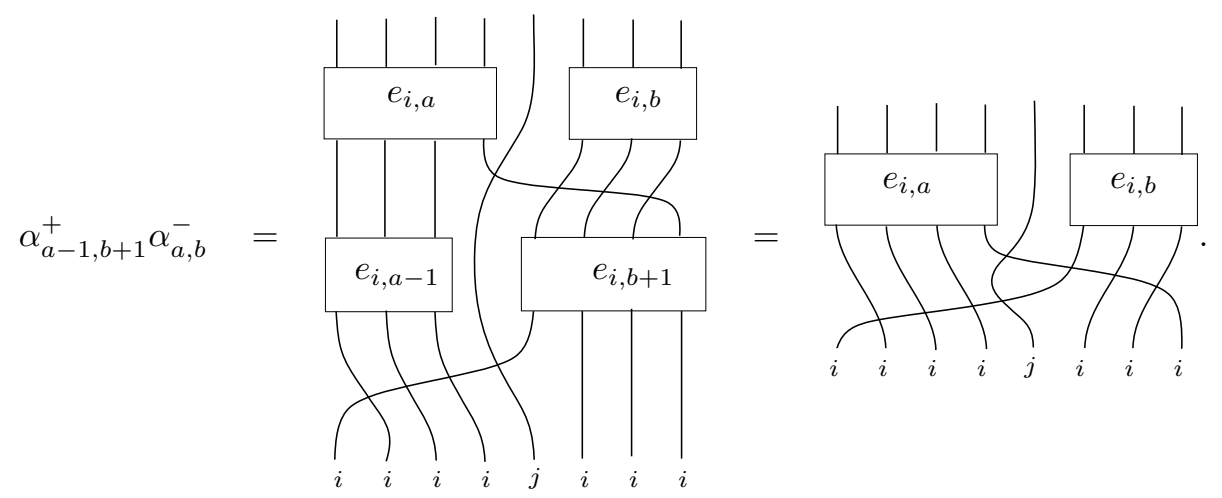

Therefore,

$$
\alpha_{a-1, b+1}^{+} \alpha_{a, b}^{-}-\alpha_{a+1, b-1}^{-} \alpha_{a, b}^{+}=(-1)^{a-1} e_{i, a} \otimes 1_{j} \otimes e_{i, b},
$$


as elements of $R(\nu)$; see below:

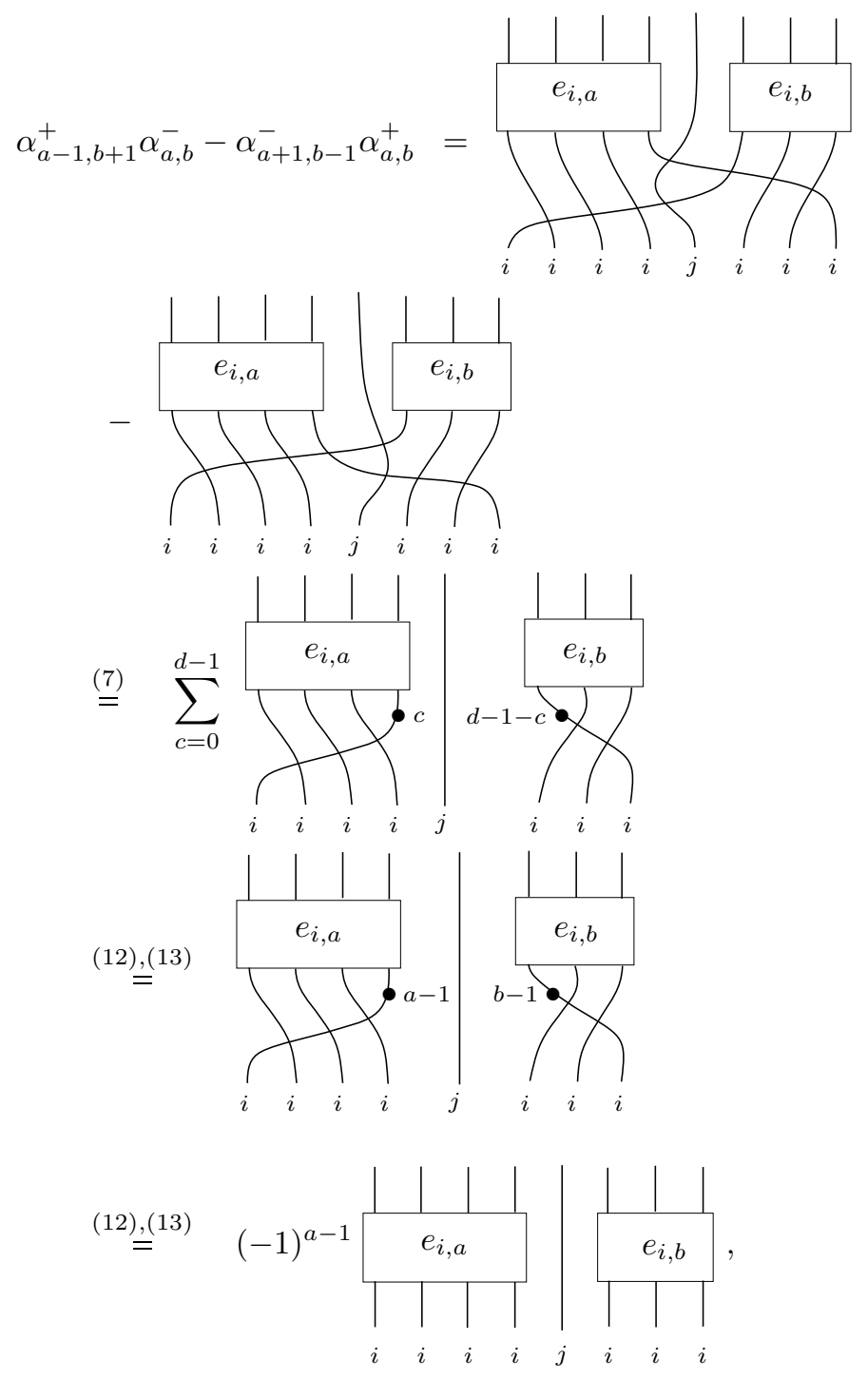

where $d=a+b-1$. Consequently,

$$
\alpha_{(a-1, b+1)}^{+} \alpha_{(a, b)}^{-}-\alpha_{(a+1, b-1)}^{-} \alpha_{(a, b)}^{+}=(-1)^{a-1} \cdot \mathrm{Id},
$$

as endomorphisms of the projective module ${ }_{(a, b)} P$, since $e_{i, a} \otimes 1_{j} \otimes e_{i, b}$ acts by the identity on ${ }_{(a, b)} P$. 
Likewise,

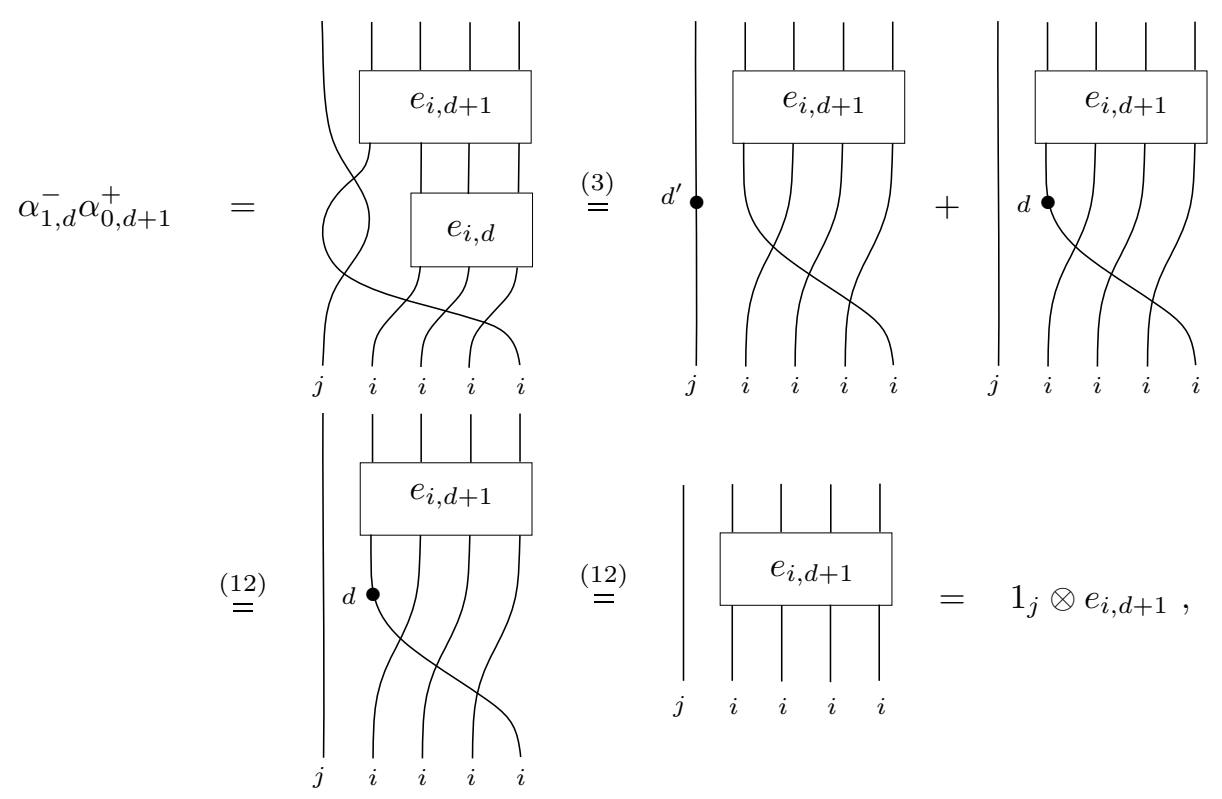

where $d^{\prime}=d_{j i}$, and

$$
\alpha_{(1, d)}^{-} \alpha_{(0, d+1)}^{+}=\mathrm{Id}
$$

as endomorphisms of ${ }_{(0, d+1)} P$. A similar computation shows that

$$
\alpha_{d, 1}^{+} \alpha_{d+1,0}^{-}=(-1)^{d} e_{i, d+1} \otimes 1_{j}
$$

as elements of $R(\nu)$, and

$$
\alpha_{(d, 1)}^{+} \alpha_{(d+1,0)}^{-}=(-1)^{d} \cdot \mathrm{Id},
$$

as endomorphisms of $(d+1,0) P$. Moreover,

$$
\alpha_{a+1, b-1}^{+} \alpha_{a, b}^{+}=0 \quad \text { and } \quad \alpha_{a-1, b+1}^{-} \alpha_{a, b}^{-}=0 .
$$

Proposition 6. For each $i, j \in I, i \neq j$ there are isomorphisms of graded right projective modules

$$
\bigoplus_{a=0}^{\left\lfloor\frac{d+1}{2}\right\rfloor} \ldots i^{(2 a)} j i^{(d+1-2 a)} \ldots P \cong \bigoplus_{a=0}^{\left\lfloor\frac{d}{2}\right\rfloor} \ldots i^{(2 a+1)} j i^{(d-2 a)} \ldots P .
$$

Proof. When $i \cdot j=0$, the isomorphism reads

$$
\ldots j i \ldots P \cong \ldots i j \ldots P
$$

and is given by left multiplication by the $i j$ intersection. When $i \cdot j<0$, earlier computations show that the maps

$$
\bigoplus_{a=0}^{\left\lfloor\frac{d+1}{2}\right\rfloor}(2 a, d+1-2 a) P \underset{\alpha^{\prime \prime}}{\longleftarrow} \bigoplus_{a=0}^{\left\lfloor\frac{d}{2}\right\rfloor}(2 a+1, d-2 a) P
$$


given by

$$
\begin{aligned}
\alpha^{\prime} & =\sum_{a=0}^{\left\lfloor\frac{d}{2}\right\rfloor} \alpha_{(2 a, d+1-2 a)}^{+}+\sum_{a=0}^{\left\lfloor\frac{d+1}{2}\right\rfloor} \alpha_{(2 a, d+1-2 a)}^{-}, \\
\alpha^{\prime \prime} & =\sum_{a=0}^{\left\lfloor\frac{d+1}{2}\right\rfloor} \alpha_{(2 a+1, d-2 a)}^{-}-\sum_{a=0}^{\left\lfloor\frac{d}{2}\right\rfloor} \alpha_{2 a+1, d-2 a}^{+},
\end{aligned}
$$

are mutually-inverse isomorphisms, implying the proposition. Maps $\alpha^{\prime}, \alpha^{\prime \prime}$ together are given by summing over all arrows in the diagram (24), with every fourth arrow appearing with the minus sign.

Corollary 7. For each $i, j \in I, i \neq j$ there are isomorphisms of graded left projective modules

$$
\bigoplus_{a=0}^{\left\lfloor\frac{d+1}{2}\right\rfloor} P_{\ldots i(2 a)} j i^{(d+1-2 a)} \ldots \cong \bigoplus_{a=0}^{\left\lfloor\frac{d}{2}\right\rfloor} P_{\ldots i^{(2 a+1)} j i^{(d-2 a)} \ldots} .
$$

Proposition [6] and Corollary 7 generalize Proposition 2.13 in [4] and can be considered a categorification of the quantum Serre relations. Corollaries 2.14 and 2.15 of [4], establishing quantum Serre relations for the characters of any $M \in$ $R(\nu)$ - mod, generalize to an arbitrary Cartan datum in the same way.

Grothendieck group as the quantum group. Induction and restriction functors for inclusions $R(\nu) \otimes R\left(\nu^{\prime}\right) \subset R\left(\nu+\nu^{\prime}\right)$ turn $K_{0}(R)$ into a twisted bialgebra, and all results of [4, Section 2.6] remain valid for an arbitrary Cartan datum. As in [4. Section 3.1] we define a homomorphism of twisted bialgebras

$$
\gamma:{ }_{\mathcal{A}} \mathbf{f} \longrightarrow K_{0}(R)
$$

which takes the product of divided powers $\theta_{i}=\theta_{i_{1}}^{\left(n_{1}\right)} \ldots \theta_{i_{r}}^{\left(n_{r}\right)}$ to $\left[P_{i}\right]$, where $\boldsymbol{i}=$ $i_{i_{1}}^{\left(n_{1}\right)} \ldots i_{i_{r}}^{\left(n_{r}\right)}$. Homomorphism $\gamma$ intertwines the bilinear forms on ${ }_{\mathcal{A}} \mathbf{f}$ and $K_{0}(R)$,

$$
(x, y)=(\gamma(x), \gamma(y)), \quad x, y \in{ }_{\mathcal{A}} \mathbf{f} .
$$

Due to the quantum Gabber-Kac theorem, this homomorphism is injective. Surjectivity of $\gamma$ follows from the arguments identical to those given in [4, Section 3.2], which, in turn, were adopted from [5, Section 5]. Alternatively, the arguments could be adopted from [2] and [9]; we settled on using a single source. We obtain

Theorem 8. $\gamma:{ }_{\mathcal{A}} \mathbf{f} \longrightarrow K_{0}(R)$ is an isomorphism of $\mathbb{N}[I]$-graded twisted bialgebras.

This theorem holds without any restrictions on the Cartan datum and on the ground field $\mathbb{k}$ over which $R(\nu)$ is defined. All other results and observations of Sections 3.2 and 3.3 of [4] extend to the general case as well. The cyclotomic quotients of $R(\nu)$, described in [4, Section 3.4], generalize to an arbitrary Cartan datum. 
It would be interesting to relate our construction to Lusztig's geometric realization of $U^{-}$in the non-simply laced case [6] and to Brundan-Kleshchev's categorification [1], [5] of $U_{q=1}^{-}$in the affine Dynkin case $A_{n}^{(2)}$.

A multi-grading. For every pair $(i, j)$ of distinct vertices of $\Gamma$, algebras $R(\nu)$ can be equipped with an additional grading, by assigning degrees -1 and 1 to the $i j$ and $j i$ crossings, respectively,

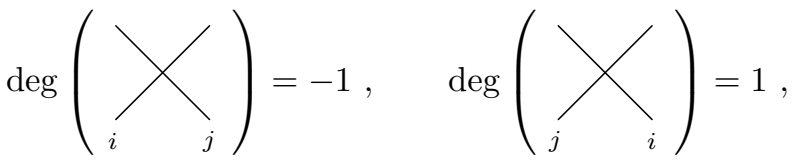

and degree 0 to all other diagrammatic generators of $R(\nu)$. These gradings are independent, and together with the principal grading, introduced above, make $R(\nu)$ into a multi-graded ring (with $\frac{n(n-1)}{2}+1$ independent gradings where $n=|\operatorname{Supp}(\nu)|$ ). The direct sum of the categories of multi-graded finitely-generated projective left $R(\nu)$-modules, over all $\nu \in \mathbb{N}[I]$, categorifies a multi-parameter deformation [7, 8] of the quantum universal enveloping algebra $U^{-}$, the quotient of the free associative algebra on $\theta_{i}, i \in I$, by the relations

$$
\sum_{a+b=d_{i j}+1}(-1)^{a} q_{i j}^{a} \theta_{i}^{(a)} \theta_{j} \theta_{i}^{(b)}=0,
$$

where $q_{i j}$ are formal variables subject to conditions $q_{i j} q_{j i}=1$.

Modifications in the simply-laced case. This section explains how to deform algebras $R(\nu)$ in the simply-laced case so that the main results of [4] will hold for the modified algebras. These deformations can be non-trivial only when the graph has cycles. As in [4, we start with an unoriented graph $\Gamma$ without loops and multiple edges. Next, fix an orientation of each edge of $\Gamma$, work over a base field $\mathbb{k}$, and, for each oriented edge $i \longrightarrow j$, choose two invertible elements $\tau_{i j}$ and $\tau_{j i}$ in $\mathbb{k}$. Denote such a datum \{orientations, invertible elements\} by $\tau$.

For each $\nu \in \mathbb{N}[I]$ consider $\mathbb{k}$-vector space $\mathcal{P} o \ell_{\nu}$ defined as in [4]. This space is the sum of polynomial rings in $|\nu|$ variables, over all sequences in $\operatorname{Seq}(\nu)$. Define $R_{\tau}(\nu)$ to be the endomorphism algebra of $\mathcal{P} o \ell_{\nu}$ generated by the endomorphisms $1_{i}, x_{k, i}, \delta_{k, i}$, over all possible $k$ and $i$, with the action as in [4, Section 2.3], with the only difference being the action of $\delta_{k, i}$ in the last of the four cases:

$$
f \mapsto\left(\tau_{i_{k} i_{k+1}} x_{k+1}\left(s_{k} \boldsymbol{i}\right)-\tau_{i_{k+1} i_{k}} x_{k}\left(s_{k} \boldsymbol{i}\right)\right)\left(s_{k} f\right) \quad \text { if } \quad i_{k} \longrightarrow i_{k+1},
$$

instead of

$$
f \mapsto\left(x_{k}\left(s_{k} \boldsymbol{i}\right)+x_{k+1}\left(s_{k} \boldsymbol{i}\right)\right)\left(s_{k} f\right) \quad \text { if } \quad i_{k} \longrightarrow i_{k+1} .
$$


The algebra $R_{\tau}(\nu)$ has a diagrammatic description similar to that of $R(\nu)$, with the following defining relations:

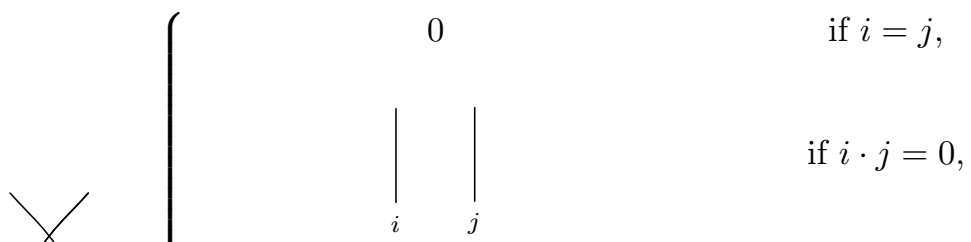

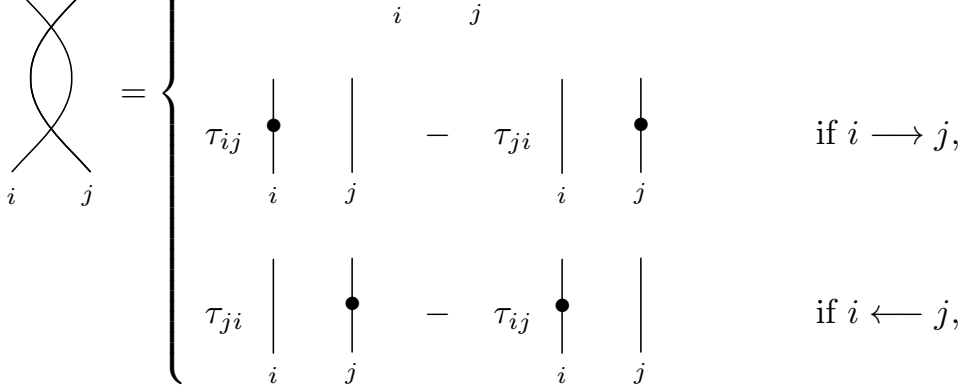
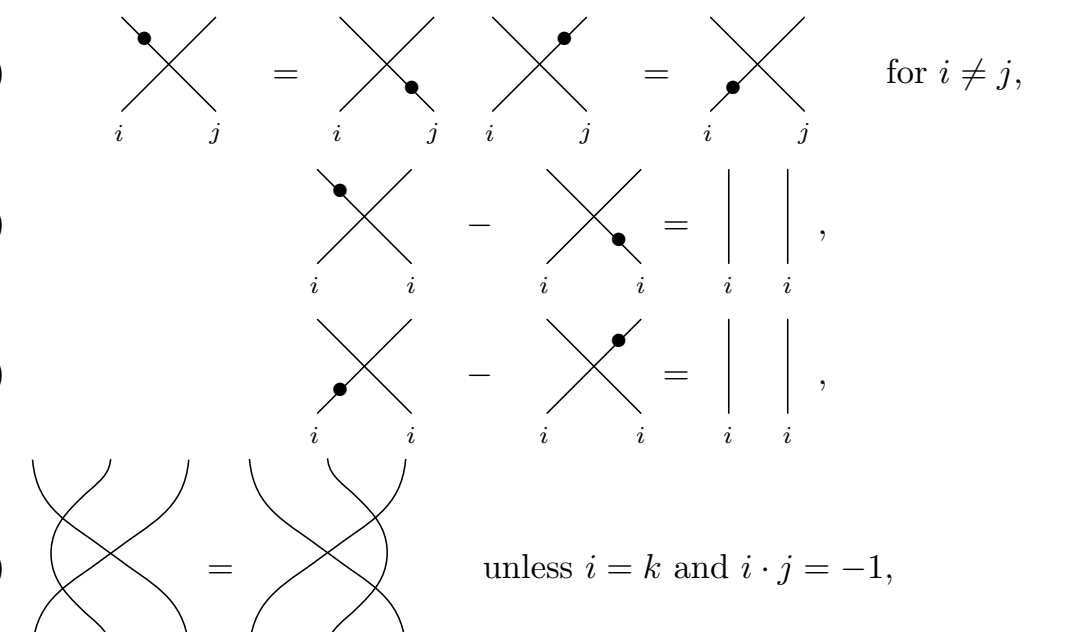

$$
\text { i }
$$

$\begin{array}{llllll}i & j & k & i & j & k\end{array}$
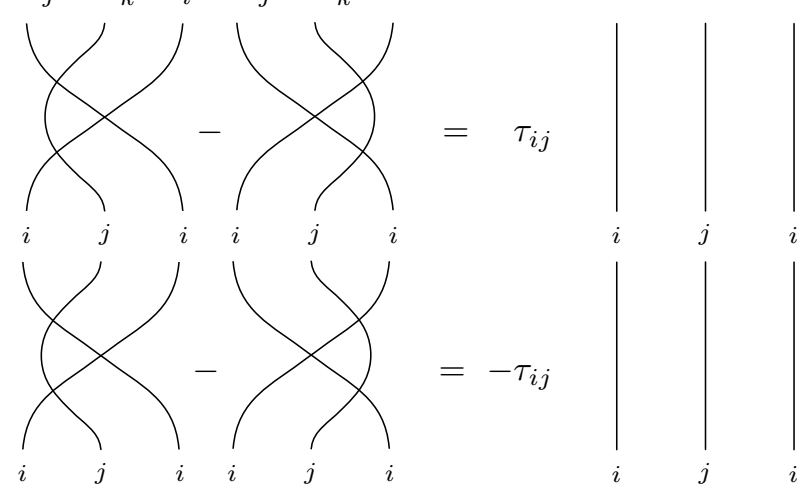

$$
\begin{aligned}
& \text { if } i \longrightarrow j, \\
& \text { if } i \longleftarrow j .
\end{aligned}
$$

Reverse the orientation of a single edge $i-j$ and change $\tau_{i j}$ to $-\tau_{i j}$ and $\tau_{j i}$ to $-\tau_{j i}$. Denote the new datum by $\tau^{\prime}$. Algebras $R_{\tau}(\nu)$ and $R_{\tau^{\prime}}(\nu)$ are isomorphic via a map which is the identity on diagrams. This way, the study of $R_{\tau}(\nu)$ reduces to the case of any preferred orientation of $\Gamma$. Rescaling one of the two possible types of the 
$i j$ crossing by $\lambda \in \mathbb{k}$ changes $\tau_{i j}$ to $\lambda \tau_{i j}$ and $\tau_{j i}$ to $\lambda \tau_{j i}$ while keeping the rest of the data fixed. We see that $R_{\tau}(\nu)$ depends only on products $\tau_{i j} \tau_{j i}^{-1}$, over all edges of $\Gamma$, via non-canonical isomorphisms. Rescalings of $i i$ crossings and dots further reduce the number of parameters to the rank of the first homology group of $\Gamma$. When graph $\Gamma$ is a forest (has no cycles), algebras $R_{\tau}(\nu)$ are all isomorphic to $R(\nu)$ via rescaling of generators. When $\Gamma$ has a single cycle, rescaling of generators reduces this family of algebras to a one-parameter family, with the parameter taking values in $\mathbb{k}^{*}$. It is likely that $R_{\tau}(\nu)$ has a description via equivariant convolution algebras in Lusztig's geometrization [6] of $U^{-}$when all $\tau_{i j}=1$ (compare with Conjecture 1.2 in [4]).

Form

$$
R_{\tau}=\bigoplus_{\nu \in \mathbb{N}[I]} R_{\tau}(\nu)
$$

The Grothendieck group $K_{0}\left(R_{\tau}\right)$ of the category of finitely-generated graded left projective modules can be naturally identified with the integral version ${ }_{\mathcal{A}} \mathbf{f}$ of $U^{-}$. All other essential constructions and results of 4 generalize from $R(\nu)$ to algebras $R_{\tau}(\nu)$ in a straightforward fashion.

Modifications in the general case. Rings $R(\nu)$ associated to an arbitrary Cartan datum admit similar modifications that depend on choosing an orientation of $\Gamma$ and invertible elements $\tau_{i j}, \tau_{j i}$ of the ground field $\mathbb{k}$ for each oriented edge $i \longrightarrow j$. The key point is the change in the definition of the endomorphism algebra, making $\delta_{k, i}$ act by

$$
f \mapsto\left(\tau_{i_{k} i_{k+1}} x_{k+1}\left(s_{k} \boldsymbol{i}\right)^{d^{\prime}}-\tau_{i_{k+1} i_{k}} x_{k}\left(s_{k} \boldsymbol{i}\right)^{d}\right)\left(s_{k} f\right) \quad \text { if } \quad i_{k} \longrightarrow i_{k+1}
$$

in the last of the four cases, with $d=d_{i_{k+1} i_{k}}$ and $d^{\prime}=d_{i_{k} i_{k+1}}$. Our proof of categorified quantum Serre relations for $R(\nu)$ requires only minor changes in the general case of $R_{\tau}(\nu)$. Everything else generalizes as well.

\section{REFERENCES}

[1] J. Brundan and A. Kleshchev. Hecke-Clifford superalgebras, crystals of type $A_{2 l}^{(2)}$ and modular branching rules for $\widehat{S}_{n}$. Represent. Theory, 5:317-403 (electronic), 2001, math.RT/0103060. MR:1870595 (2002j:17024)

[2] I. Grojnowski and M. Vazirani. Strong multiplicity one theorems for affine Hecke algebras of type A. Transform. Groups, 6(2):143-155, 2001. MR1835669 (2002c:20008)

[3] L. Kauffman and S. Lins. Temperley-Lieb recoupling theory and invariants of 3-manifolds, volume 134 of Annals of Mathematics Studies. Princeton University Press, 1994. MR 1280463 (95c:57027)

[4] M. Khovanov and A. Lauda. A diagrammatic approach to categorification of quantum groups I. Represenation Theory, 13:309-347 (electronic), 2009. MR2525917 (2010i:17023)

[5] A. Kleshchev. Linear and projective representations of symmetric groups, volume 163 of Cambridge Tracts in Mathematics. Cambridge Univ. Press, 2005. MR2165457|(2007b:20022)

[6] G. Lusztig. Introduction to quantum groups, volume 110 of Progress in Mathematics. Birkhäuser Boston Inc., Boston, MA, 1993. MR1227098 (94m:17016)

[7] M. Okado and H. Yamane. $R$-matrices with gauge parameters and multi-parameter quantized enveloping algebras. In Special functions (Okayama, 1990), ICM-90 Satell. Conf. Proc., pages 289-293. Springer, Tokyo, 1991. MR1166822(93f:17025) 
[8] N. Reshetikhin. Multiparameter quantum groups and twisted quasitriangular Hopf algebras. Lett. Math. Phys., 20(4):331-335, 1990. MR1077966 (91k:17012)

[9] M. Vazirani. Irreducible modules over the affine Hecke algebra: A strong multiplicity one result. Ph.D. thesis, UC Berkeley, 1999, math.RT/0107052.

Department of Mathematics, Columbia University, New York, New York 10027

E-mail address: khovanov@math.columbia.edu

Department of Mathematics, Columbia University, New York, New York 10027

E-mail address: lauda@math.columbia.edu 